Study/Objective: Assessment of Critical Gaps in Aid Worker Preparation during the 2014 Ebola Outbreak.

Background: During the author's time as a clinician and clinical instructor during the recent Ebola emergency response, two mambas were found in Ebola Treatment Units (ETUs). In addition to the ETU setting, the very nature of addressing Ebola necessitates fieldwork in jungle conditions. Each of the three main countries affected by Ebola have a significant number of venomous reptiles, as well as other potential plant and animal sources of toxin exposures. The author worked independently for six weeks with Liberian Ministry of Health $(\mathrm{MoH})$ and the World Health Organization $(\mathrm{WHO})$ as a Facilitator for Ebola Phase III "Hot" Training.

Additionally, he assisted in the preparation and opening of a 50 bed Ebola Treatment Unit (ETU), where he was also responsible for providing clinical care, comprehensive education and training, and oversaw field operations of three ambulances and associated personnel. At no point did any of the formal predeployment training address the potential for envenomation from the local flora and fauna. I believe this is a critical lack that should be addressed in future responses.

Methods: Comprehensive review of CDC, WHO, US Military, and aid organization Ebola training materials. Informal verbal surveys conducting as an instructor during the final phase of Ebola hot zone training.

Results: Despite the potential of a lethal envenomation, no pre-deployment coursework included material addressing this possibility. Furthermore, informal surveys of hundreds of Ebola emergency response workers representing dozens of aid agencies revealed that not one organization had prepared for an envenomation incident.

Conclusion: Despite the fieldwork that is inherent in an Ebola emergency response, there were no plans in place regarding antivenin or medical evacuation from the field. The entire focus was on preventing exposure to Ebola. This is analogous to a "distracting mechanism of injury" in Emergency Medicine and Trauma, in which tunnel vision impairs a comprehensive survey.

Conclusions: When there is a probability of encountering envenomation during fieldwork, especially potentially lethal envenomation, pre-deployment training should include both a comprehensive risk assessment; as well as appropriate contingency plans. Failing to plan is planning to fail.

Prehosp Disaster Med 2017;32(Suppl. 1):s121-s122

doi:10.1017/S1049023X17003454

\section{The Fatal Fruit: A Cautionary Tale in Situational}

\section{Awareness}

Sean Smith

Critical-care Professionals International, Critical-Care Professionals International, Durham/United States of America

Study/Objective: Emphasize Need for Medical Mission teams to familiarize themselves with local flora/ fauna and potential intoxications/ envenomations.

Background: A four-year-old girl presented to our Haitian hospital with profound hypoglycemia and a six-hour history of seizures. Initially, aside from addressing the hypoglycemia and administering benzodiazepines, the staff was unable to provide definitive diagnosis or treatment. Subsequent in depth interviews with the parents via translators allowed the staff to determine that ingestion of unripe ackee fruit (Blighia sapida) was the probable cause of the child's symptoms.

Methods: Case Study and review of physiology, biochemistry, and management of ackee fruit toxicity.

Results: Increased readiness on the part of healthcare providers to recognize and treat Ackee Fruit Toxicity, as well as increased situational awareness regarding potential exposures outside their cultural norms.

Conclusion: Discussion: The inability to obtain a complete history and physical (as is often the case in toxic ingestions), as well as a lack of familiarity with local toxic plants potentially led to a delay in definitive treatment. This is particularly problematic when medical staff is deploying to unfamiliar regions. Conclusions: Medical Mission workers should educate themselves as to local specific toxins that they may lack familiarity with. Delays in diagnosis results in delays in comprehensive care, with potential subsequent increases in morbidity and mortality.

Failing to Plan is Planning to Fail.

Prehosp Disaster Med 2017;32(Suppl. 1):s122

doi:10.1017/S1049023X17003466

\section{When a Little Human is Bitten Twice by a Large}

Venomous Snake: The Providers Disagree with the Original Consultant Recommendation

Benjamin N. Abo, Spencer J. Johnson, David A. Kranc

Emergency Medicine, University of Florida, Gainesville/FL/United States of America

Study/Objective: This is a case of a severely envenomed child by not one, but two bites from a confirmed large adult Florida coral snake (Micrurus fulvous), that exhibited with immediate systemic manifestations. Initial consultation from a wilderness medicine and emergency medicine specialist, suggested one treatment, but consultation with a toxicology service asking certain questions gave conflicting recommendations to treatment. Bringing in other experienced consultants and advocating for the patient, led to the change of recommendation by the initial consultant; and finally treatment, though delayed initially, of the patient with the appropriate antivenom.

Background: Literature is scant at best on how often even medical professionals / attending physicians might disagree with consulting specialists. However, it is important for any medical professional at any level, to be able to advocate for what might be best for the patient, as well as to educate the patient or their caretakers. This, of course, can be done civilly and professionally, although this is a skillset seldom taught. Elapid snake envenoming, specifically coral snake envenoming, requires important education to determine if and how much antivenom, the only true cure for venom, might be needed. Many online resources are incorrect or incomplete with regards to the proper treatment of snakebites, and possibly even harmful.

Methods: This is a case of a double envenoming of a young child by a Florida coral snake, leading to severe systemic effects 
that for various reasons had a significant delay in treatment with antivenom. This case will be used to highlight important aspects to snake envenomation and recommendations on dealing with consultants.

Results: In the end, our young patient was ultimately discharged from the Intensive Care Unit (ICU) with significantly improved central and peripheral nervous system symptoms.

Conclusion: Conflicting treatments and patient advocacy need to be carefully balanced, and even disagreements can be handled professionally.

Prehosp Disaster Med 2017;32(Suppl. 1):s122-s123

doi:10.1017/S1049023X17003478

Using the Epidemic Curve to Inform Social and Behavior Change at Scale During Epidemic Response.

Amanda Mcclelland

International Federation of the Red Cross and Red Crescent, Geneva/ Switzerland

Study/Objective: The International Federation of the Red Cross and Red Crescent (IFRC) presents a model for Social Behavior Change in emergency contexts that supports local actors in low resources settings, and engage with communities and utilize the epidemic curve to help inform response. Using the epidemic curve at a granular level, allows national communication plans to be tailored in time and place in relation to the movement of the epidemic, ensuring messaging and activities are tailored to where and when communities are in the epidemic evolution and combines with local context

Background: The focus on community engagement and the role of 'Social and Behavior Change' (SBC) during development and emergency interventions is not new. Much work has been done in this area with a plethora of theories and models to support implementation across health topics and sectors, as diverse as obesity and STD prevention. These models, often based on social science, psychology and social marketing have a commonality that includes triggering motivation for change, supporting and maintaining the new behavior. They rely heavily on in depth assessments of root causes of the behavior, cultural contexts and reflective program design. However, how do these models interface in an epidemic, where time and resources can be limited, the motivation for change is often clear, the threat time limited and moving geographically?

Methods: The IFRC supports an average of 20 public health threats a year. Providing quality SBC programming at scale in low resources settings, remain a significant challenge, however new models of implementation are being field tested.

Results: A new model was developed.

Conclusion: Providing a clear link between the epidemic evolution in time, place and person allows specific targeting of interventions to support prevention, reduction and eradication of transmission to at risk groups. The combination of the improved utilization of social science to inform programming, needs to be a two-way dialogue, where epidemiological data is used to target and tailor SBC.

Prehosp Disaster Med 2017;32(Suppl. 1):s123

doi:10.1017/S1049023X1700348X
Nutrition Centers in Protracted Crisis Context: Field Study from Syria Haytham Qosa ${ }^{1}$, Hazem Bakleh ${ }^{2}$, Salim Sohani ${ }^{1}$, Bernice Tiggelaar ${ }^{1}$, Maggie Almously ${ }^{2}$

1. Global Health Unit, Canadian Red Cross, Ottawa/Canada

2. Syrian Arab Red Crescent, Damascus/Syria

Study/Objective: The objective of this field study is to examine the effectiveness of implementing comprehensive nutritional services at the community level in the complex humanitarian crisis in Syria; and to disseminate this knowledge among other humanitarian actors.

Background: Syria is one of the largest and most complex crises, experienced by the international humanitarian system. Difficulties of access and transportation of goods have increased the prices and reduced the availability of commodities. Cases of Severe Acute Malnutrition (SAM) and Moderate Acute Malnutrition (MAM) - both components of Global Acute Malnutrition (GAM), are on the rise in Syria. The Syrian Arab Red Crescent in cooperation with Canadian Red Cross are supporting 6 nutrition centers in Hama, Salamiyeh, Al Tal, Tartous, Aleppo and Swaida.

Methods: In Syria, the Community-based Management of Acute Malnutrition (CMAM) approach targets of acutely malnourished children under five, and pregnant and lactating women through community outreach, Supplementary Feeding Program (SFP) and Outpatient Therapeutic Program (OTP). The cases either come directly to the centers or referred by doctors or outreach mobile teams who conduct door-to-door nutritional assessments, using Mid-Upper Arm Circumference (MUAC) measurements. Quantitative and qualitative data are collected and analyzed on regular bases.

Results: Until the end of October 2016 the centers were able, collectively, to screen a total of 38,847 children and 8,434 pregnant and lactating women. We've identified and treated total of 254 SAM cases and 1,574 MAM cases amongst children, and 1,167 cases of MAM among pregnant and lactating women. With ensuring humanitarian principles are always respected, we consider various tactics to overcome evolving challenges that may include access, security, patients and family compliance and disruption of nutritional supplements.

Conclusion: Implementation knowledge generated from this project can model challenges and solutions in comprehensive nutritional services at the community level in complex humanitarian crisis.

Prehosp Disaster Med 2017;32(Suppl. 1):s123

doi:10.1017/S1049023X17003491

Applying the Experience of Level-2 Military Surgical Teams to Disaster Medicine

Przemyslaw W. Gula, Bartosz Zakowski, Witold Bronowski

Polish Armed Forces, Krakow/Poland

Study/Objective: The objective of the paper is to assess the options of using the experience of level-2 military surgical teams operating in military conflict in the context of treating mass casualty and disaster victims. 